\title{
Quantification of Nuclear Data and Manufacturing Uncertainties in VERA
}

\author{
Christopher Sedota, Scott Palmtag \\ North Carolina State University \\ Raleigh, NC 27607 \\ cssedota@ncsu.edu,sppalmta@ncsu.edu
}

\begin{abstract}
Uncertainty quantification (UQ) was performed using the Consortium for the Advanced Simulation of Light Water Reactors (CASL) multiphysics core simulator VERA. Typically, only nuclear data cross sections are considered when trying to obtain uncertainty information in reactor simulation applications. In this paper, uncertainty in both nuclear cross section data and fuel manufacturing processes is analyzed using VERA. Uncertainties due to cross sections was determined by generating one thousand perturbed cross section libraries using the cross section covariance data provided in the evaluated nuclear data library. Uncertainty due to manufacturing was also determined using stochastic sampling and VERA. The use of similar stochastic sampling techniques for the same problems allows for the direct comparison of uncertainty stemming from the two sources of uncertainty. Sample size is considered due to the potentially large computational cost of stochastic sampling techniques, as is demonstrated in a full core depletion. It was found that for the Pressurized Water Reactor (PWR) pincell case at Hot Zero Power (HZP), the standard deviation in the neutron multiplication factor produced by material uncertainty was $101 \mathrm{pcm}$, while the standard deviation in the neutron multiplication factor produced by cross section uncertainty was $730 \mathrm{pcm}$. While the uncertainty in neutron multiplication factor due to cross section uncertainty is larger than uncertainty due to manufacturing uncertainties, neglecting manufacturing uncertainties may be an unacceptable oversight in certain high-precision simulation applications.
\end{abstract}

KEYWORDS: Uncertainty Quantification, VERA, Core Simulator

\section{INTRODUCTION}

Uncertainty is inherent in any real system. While uncertainty is sometimes neglected when modeling systems, its quantification is extremely important. If uncertainty can be accurately estimated, greater confidence can be placed in simulated results. Having greater confidence in the validity of simulated results is hugely beneficial, as non-simulated experimental results are extremely expensive to obtain. In this project, uncertainties in cross sections and material parameters will be propagated through the Consortium for the Advanced Simulation of Light Water Reactors' (CASL) code system, the Virtual Environment for Reactor Applications (VERA). Stochastic sampling was used to obtain realizations of uncertainties due to cross section data and manufacturing uncertainties. While stochastic sampling schemes have their downsides, such as the difficulty in identifying uncertainty due to individual parameters and sometimes excessive computational cost, their benefits are significant. Due to the noninvasive nature of stochastic sampling, computer codes can be used simply as models to propagate uncertainty which is particularly beneficial when performing multiphysics simulations. 


\section{METHODOLOGY}

\subsection{Neutronics Solver}

MPACT [1], the primary neutronics solver in VERA, was used as the model in this study. MPACT is a three-dimensional transport solver which uses the 2D/1D methodology. Each radial plane in the core is solved using the Method of Characteristics (MOC), and radial calculations are coupled to 1D transport solutions for each fuel rod. The effective neutron multiplication factor (k-eff) calculated by MPACT is the model response. MPACT cross sections were generated using a 51 energy group library based on ENDF/B-VII.1 nuclear data. Resonance parameters are calculated using the subgroup method.

\subsection{Cross Section Uncertainties}

Stochastic sampling was used to represent the uncertainty in cross sections. In the stochastic sampling method, mean cross section values are perturbed using covariance data to generate realizations of cross section libraries. In this project, the Sampler Tool in SCALE [2] was used to generate one thousand unique cross section libraries. Each of these libraries were then used in MPACT for each of the problem configurations to estimate the resultant uncertainty in k-eff.

\subsection{Manufacturing Uncertainties}

The effect of manufacturing uncertainties was also analyzed in this paper. Mean values and uncertainties in five material parameters were taken from the UAM-LWR benchmark [3]. To this point, only the PWR Pincell at HZP has been examined using stochastic sampling. The five material parameters were each sampled individually while fixing the other parameters to their mean values in order to examine the uncertainty associated with each parameter. Additionally, all parameters were varied simultaneously to examine the uncertainty associated with all material parameters. The five material parameters, their mean values, and their $3 \sigma$ uncertainties are listed in Table I.

Table I. Material parameters: Mean value and uncertainty associated with five material parameters.

\begin{tabular}{|c|c|c|}
\hline Parameter & Mean Value & $3 \sigma$ Uncertainty \\
\hline Fuel Density & $10.283 \mathrm{~g} / \mathrm{cm}^{3}$ & $\pm 0.17 \mathrm{~g} / \mathrm{cm}^{3}$ \\
\hline Pellet Diameter & $9.391 \mathrm{~mm}$ & $\pm 0.013 \mathrm{~mm}$ \\
\hline Gap Thickness & $0.0955 \mathrm{~mm}$ & $\pm 0.024 \mathrm{~mm}$ \\
\hline Clad Thickness & $0.673 \mathrm{~mm}$ & $\pm 0.025 \mathrm{~mm}$ \\
\hline Fuel Enrichment & $4.85 \mathrm{w} / \mathrm{o}$ & $\pm 0.00224 \mathrm{w} / \mathrm{o}$ \\
\hline
\end{tabular}

\section{CROSS SECTION UNCERTAINTY RESULTS}

Several test problems were taken from the UAM-LWR benchmark [3]. Pressurized Water Reactor (PWR) and Boiling Water Reactor (BWR) Pincells were analyzed at both Hot Zero Power (HZP) and Hot Full Power (HFP). In addition, two-dimensional PWR and BWR lattices at HZP and HFP were examined with the control rods fully withdrawn (unrodded) and fully inserted (rodded). Finally, a simple twodimensional PWR colorset was analyzed at HZP and HFP. This set of test problems covers a range of configurations without being prohibitively expensive to run. 


\subsection{Pincell Cases}

A summary of the results for the pincell cases is shown in Table II. The benchmark average results [4] were calculated by the UAM benchmark team and are simply the average of all the submitted results for each case. The uncertainty data is reported as a relative standard deviation, which is the standard deviation normalized by the k-eff for each case.

Table II. Pincell results: Effective neutron multiplication factor with uncertainty calculated by MPACT and from benchmark.

\begin{tabular}{|c|c|c|}
\hline Case & MPACT Result & $\begin{array}{c}\text { Benchmark } \\
\text { Average }\end{array}$ \\
\hline BWR HZP & $1.34476 \pm 0.552 \%$ & $1.3408 \pm 0.603 \%$ \\
\hline BWR HFP & $1.22976 \pm 0.606 \%$ & $1.2257 \pm 0.680 \%$ \\
\hline PWR HZP & $1.42756 \pm 0.511 \%$ & $1.4235 \pm 0.586 \%$ \\
\hline PWR HFP & $1.40966 \pm 0.515 \%$ & $1.4075 \pm 0.293 \%$ \\
\hline
\end{tabular}

Overall, the results from MPACT agree well with the benchmark average results. The only major discrepancy is that the uncertainty in k-eff for the PWR case at HFP is considerably lower in the benchmark average than in the MPACT results. However, the trend for these cases seems to be that the uncertainties are similar but slightly higher when comparing HFP results to HZP results. This trend is observed in the MPACT results when considering the BWR (0.606\% compared to $0.552 \%)$ and the PWR $(0.515 \%$ and $0.511 \%)$, and the benchmark average results for the BWR $(0.680 \%$ and $0.603 \%)$. The only case that breaks the trend is the benchmark average PWR results. It is believed that the low relative standard deviation in the benchmark average PWR HFP case is due to an outlier in the data collected by the benchmark, as all other cases have comparable relative standard deviations. In addition, the fact that not all participants used the same cross section library, and therefore were using different data in some cases to propagate uncertainty, certainly accounts for some of the discrepancy seen in the results.

Figure 1 shows the k-eff distribution from evaluating the BWR pincell at HZP using one thousand multigroup libraries. Other configurations show similar results.

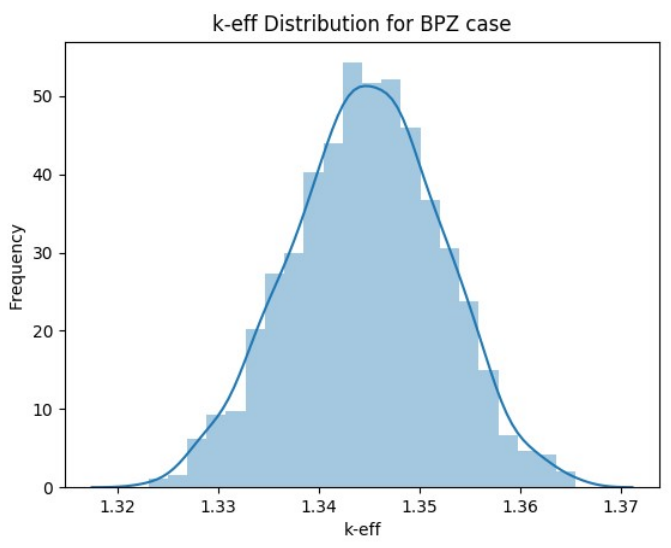

Figure 1. Multiplication Factor Distribution for the BWR Pincell at HZP (BPZ). 


\subsection{Lattice-Level Cases}

A summary of the results for the PWR lattice and colorset is shown in Table III. The results for the Rodded PWR cases and colorsets were not published in the benchmark summary document, so a direct comparison could not be made.

Table III. PWR lattice and colorset results: Effective neutron multiplication factor and uncertainty calculated by MPACT and from benchmark.

\begin{tabular}{|c|c|c|}
\hline Case & MPACT Result & $\begin{array}{c}\text { Benchmark } \\
\text { Average }\end{array}$ \\
\hline Unrodded HZP & $1.41447 \pm 0.501 \%$ & $1.4123 \pm 0.458 \%$ \\
\hline Unrodded HFP & $1.39879 \pm 0.504 \%$ & $1.3979 \pm 0.471 \%$ \\
\hline Rodded HZP & $1.07772 \pm 0.527 \%$ & N/A \\
\hline Rodded HFP & $1.06272 \pm 0.532 \%$ & N/A \\
\hline Colorset HZP & $1.38468 \pm 0.499 \%$ & N/A \\
\hline Colorset HFP & $1.36889 \pm 0.502 \%$ & N/A \\
\hline
\end{tabular}

For the unrodded PWR cases, the MPACT results compare well with the benchmark average results both in mean value and uncertainty.

A summary of the results for the BWR lattice is shown in Table IV. The only reported benchmark average results were for the unrodded case at HZP.

Table IV. BWR lattice results: Effective neutron multiplication factor and uncertainty calculated by MPACT and from benchmark.

\begin{tabular}{|c|c|c|}
\hline Case & MPACT Result & $\begin{array}{c}\text { Benchmark } \\
\text { Average }\end{array}$ \\
\hline Unrodded HZP & $1.10725 \pm 0.557 \%$ & $1.1067 \pm 0.519 \%$ \\
\hline Unrodded HFP & $1.07894 \pm 0.575 \%$ & N/A \\
\hline Rodded HZP & $0.863599 \pm 0.548 \%$ & N/A \\
\hline Rodded HFP & $0.794981 \pm 0.590 \%$ & N/A \\
\hline
\end{tabular}

While benchmark data was very limited for the two-dimensional BWR lattices, the unrodded HZP case matches the benchmark average result quite well, and the other cases are in line with what would be expected given the trends seen in the PWR lattice cases. 


\subsection{Full Core Depletion of Watts Bar Unit 1 Cycle 1}

All of the results shown have been for relatively simple problems. However, the methodology used to this point can be used to analyze much more complex problems, including those involving full multiphysics coupling. The stochastic sampling technique was applied to a full core problem - Watts Bar Unit 1 Cycle 1 [5], to examine the uncertainty in the critical boron concentration as fuel depletes. The boron letdown curves for 29 cross section libraries are shown in Figure 2. The standard deviation of the critical boron concentration was calculated and converted to the standard deviation of the neutron multiplication factor assuming a boron reactivity coefficient of $-8 \mathrm{pcm} / \mathrm{ppm}$. It was found that the standard deviation of $\mathrm{k}$-eff ranged from $333 \mathrm{pcm}$ at the beginning of the cycle to $439 \mathrm{pcm}$ near the end of the cycle, at an exposure of $410.7 \mathrm{MWd} / \mathrm{MTU}$. The uncertainty in k-eff due to cross section uncertainty in the cross sections is comparable to results obtained in the smaller PWR assembly and colorset configurations.

While all previous cases have been run with one thousand perturbed cross section libraries, the full core depletion was only run with 29 cross section libraries. The full-core case demonstrates one of the main limitations of stochastic sampling - excessive computational cost for large problems. This issue is examined in the following section.

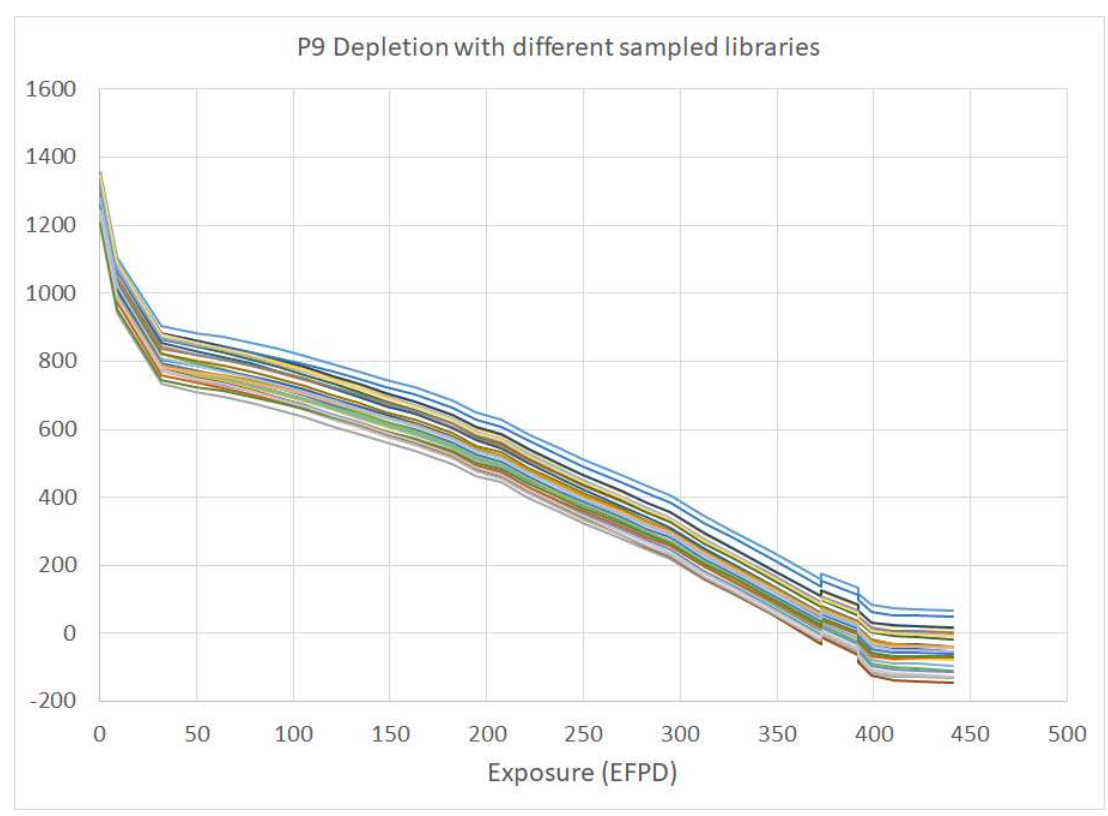

Figure 2. Full Core Depletion for Watts Bar Unit 1 Cycle 1 with Perturbed Cross Section Libraries.

\subsection{Sample Size}

An item of particular concern in our study is sample size. While running a transport solver one thousand times with one thousand unique cross section libraries to generate high quality statistical data is feasible for smaller cases such as pincells and two-dimensional lattices, it is not currently feasible for reactor-scale problems such as the Watts Bar depletion due to computational cost. The largest problem we ran with the one thousand libraries was a three-by-three two-dimensional colorset of PWR lattices, with one rodded lattice surrounded by eight unrodded lattices. Confidence intervals were constructed using the $\mathrm{t}$-distribution for the running average of k-eff for the PWR colorset at HFP, shown in Figure 3. (Note that PCF in the figure refers to PWR Colorset at HFP). 


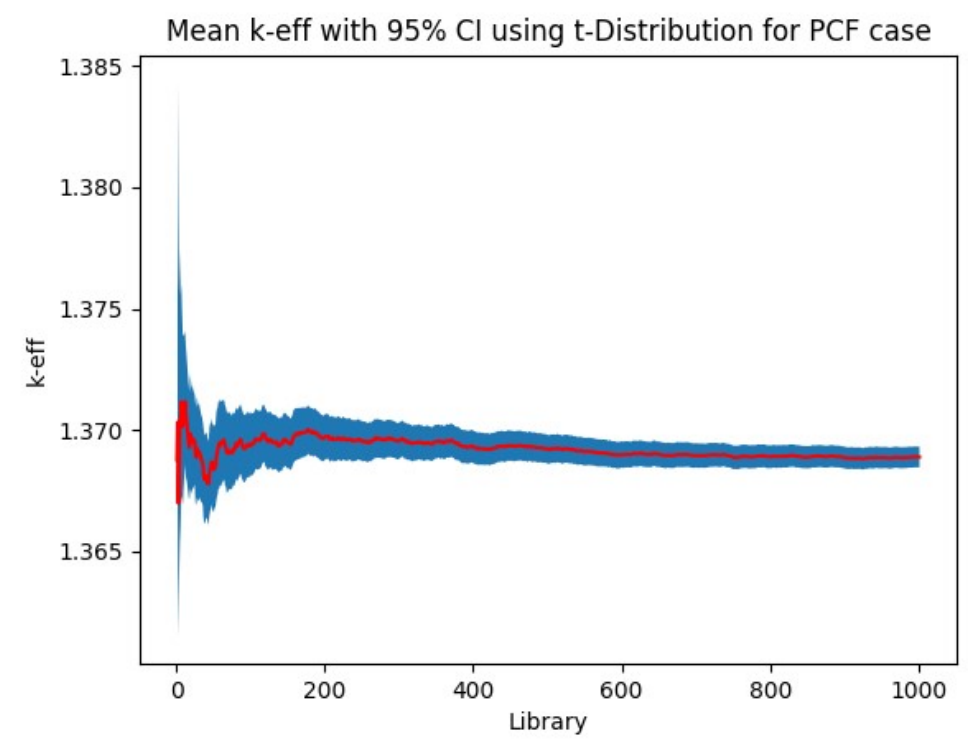

Figure 3. Confidence Intervals for Mean k-eff for PWR Colorset at HFP (PCF).

It is evident from Figure 3 that the increase in confidence gained by using one thousand libraries instead of, for instance, 500 - is marginal. The standard error of the mean is inversely proportional with sample size, so the effect of increasing sample size has diminishing returns in statistical confidence. This trend is seen in the estimate of the standard error of the mean, shown in Table V. Doubling the sample size from 500 cross section libraries to one thousand only reduces the estimated standard error of the mean by approximately $5 \mathrm{pcm}$. While 29 samples is likely too small to establish much statistical confidence in our result, this study shows that 250 libraries is likely sufficient in many cases.

Table V. Error in mean k-eff for PWR colorset at HFP : Effective neutron multiplication factor and uncertainty calculated by MPACT and from benchmark.

\begin{tabular}{|c|c|c|c|}
\hline Sample Size & Mean k-eff & $\begin{array}{c}\text { Standard } \\
\text { Deviation } \\
\text { Estimate }(\mathrm{pcm})\end{array}$ & $\begin{array}{c}\text { Standard Error of } \\
\text { Mean Estimate } \\
(\mathrm{pcm})\end{array}$ \\
\hline 29 & 1.36909 & 548 & 101.8 \\
\hline 100 & 1.36936 & 657 & 65.7 \\
\hline 250 & 1.36954 & 659 & 41.7 \\
\hline 500 & 1.36921 & 672 & 30.1 \\
\hline 1000 & 1.36889 & 674 & 24.6 \\
\hline
\end{tabular}




\section{MANUFACTURING UNCERTAINTY RESULTS}

Five parameters were varied to study the effect of manufacturing uncertainties on the effective neutron multiplication factor: fuel density, fuel pellet diameter, gap width, clad thickness, and fuel enrichment. These five parameters were varied both independently and simultaneously by stochastic sampling out of the uncertainties provided in the benchmark, and reproduced in Table I.

To this point, material uncertainties were only examined in the PWR pincell at HZP. The estimate for $\mathrm{k}$-eff for the simultaneous parameter variation was determined to be 1.4302 with a relative standard deviation of $0.071 \%$. This corresponds to a standard deviation of $101 \mathrm{pcm}$. This can be directly compared to the standard deviation when varying cross sections, which was determined to be $0.511 \%$, or $730 \mathrm{pcm}$. The uncertainty from the given material uncertainties is much lower than the uncertainty from the cross sections, but still may be significant in certain applications. A histogram of the k-eff values obtained with different manufacturing dimensions is shown in Figure 4.

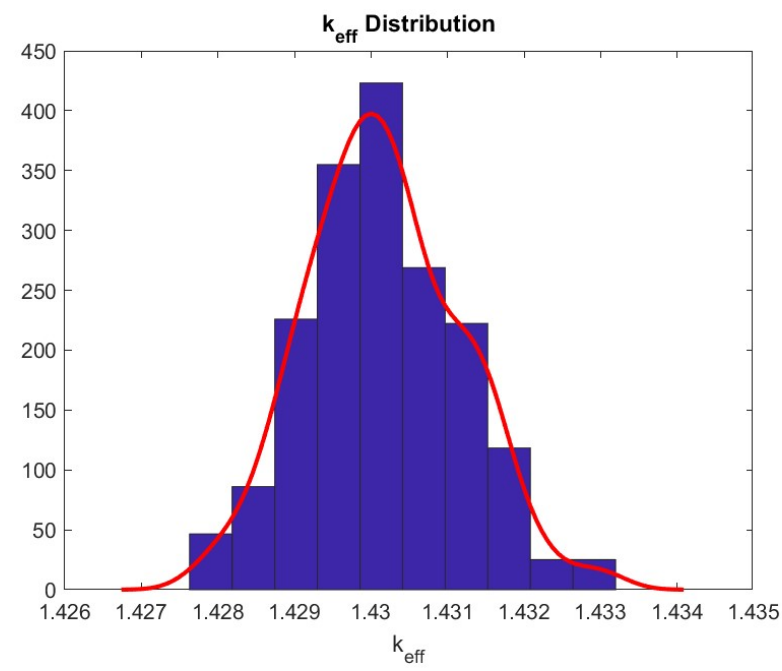

Figure 4. Multiplication Factor Distribution Varying Material Parameters.

The full results of the stochastic sampling of material parameters are shown in Table VI. The standard deviations are reported in units of percent mille $(\mathrm{pcm})$. The parameter that contributed the most uncertainty to k-eff was the cladding thickness. The fuel density, pellet diameter, gap thickness, and clad thickness uncertainties produce similar magnitudes of uncertainty in k-eff, but the U-235 enrichment is a notable outlier. The sensitivity to fuel enrichment is smaller than the other material parameters by more than a factor of ten and this is directly attributable to the low manufacturing uncertainty. 
Table VI. Material parameter uncertainty quantification: Standard deviation of the effective neutron multiplication factor when varying certain parameters.

\begin{tabular}{|c|c|}
\hline Varied Parameter & Standard Deviation $(\mathrm{pcm})$ \\
\hline Fuel Density & 59 \\
\hline Pellet Diameter & 29 \\
\hline Gap Thickness & 44 \\
\hline Clad Thickness & 67 \\
\hline Fuel Enrichment & 2 \\
\hline All Materials & 101 \\
\hline Cross Sections & 730 \\
\hline
\end{tabular}

\section{CONCLUSIONS}

VERA is a useful tool in reactor simulation. In this paper, it was shown that VERA tools can be used to perform uncertainty quantification using stochastic sampling. Uncertainty in both neutron cross sections and material parameters were considered for a wide range of test problems, and compared to benchmark results where applicable. In general, good agreement was found between the results found and results generated by others using other uncertainty quantification methods. Stochastic sampling was then used to demonstrate uncertainty quantification due to cross section uncertainties for a full-core reactor problem which has multiphysics feedback.

\section{ACKNOWLEDGMENTS}

Funding for this project was provided by the Consortium for the Advanced Simulation of Light Water Reactors (CASL), a DOE innovation hub.

\section{REFERENCES}

1. B. Kochunas et al., "VERA Core Simulator Methodology for Pressurized Water Reactor Cycle Depletion," Nucl. Sci. Eng., 185, (2017).

2. B.T. Rearden, M.A. Jessee, "SCALE Code System," Oak Ridge National Laboratory (2016).

3. K. Ivanov, M. Avramova, S. Kamerow, I. Kodeli, E. Sartori, E. Ivanov, O. Cabellos, "Benchmark for Uncertainty Analysis in Modelling (UAM) for the Design, Operation, and Safety Analysis of LWRs," NEA/NSC/DOC(2013)7, Nuclear Energy Agency (2013).

4. Ryan N. Bratton, M. Avramova, K. Ivanov, "OECD/NEA Benchmark for Uncertainty Analysis in Modeling (UAM) for LWRs - Summary and Discussion of Neutronics Cases (Phase I)," Nucl. Eng. Tech., 46, Issue 3, pp 313-342 (2014).

5. A. Godfrey, "VERA Core Physics Benchmark Progression Problem Specifications," Technical Report CASL-U-2012-0131-004, Revision 4 (2014). 